\title{
EARLY DETECTION OF PLEURO-PULMONARY TUBERCOLOSIS BY BEDSIDE LUNG ULTRASOUND: A CASE REPORT AND REVIEW OF LITERATURE
}

\author{
Andrea Boccatonda ${ }^{1}$, giulio cocco ${ }^{2}$, Antonio Corvino ${ }^{3}$, Ilaria Rossi ${ }^{2}$, Andrea Delli Pizzi ${ }^{2}$, \\ Claudio Ucciferri ${ }^{2}$, Vecchiet J. ${ }^{4}$, Falasca $\mathrm{K}^{4}$, and D'Ardes D. ${ }^{2}$ \\ ${ }^{1}$ Bentivoglio Hospital \\ ${ }^{2}$ Gabriele d'Annunzio University of Chieti and Pescara Department of Sciences \\ ${ }^{3}$ Federico II University Hospital \\ ${ }^{4}$ Gabriele d'Annunzio University of Chieti and Pescara
}

February 8, 2022

\begin{abstract}
We present a case in which lung Ultrasound was relevant to reach an early diagnosis of lung tuberculosis and to manage the patient in the right setting. Moreover, ultrasound allowed to detect and treat massive pleural effusion through an ultrasoundguided thoracentesis.

\section{EARLY DETECTION OF PLEURO-PULMONARY TUBERCOLOSIS BY BEDSIDE LUNG ULTRASOUND: A CASE REPORT AND REVIEW OF LITERATURE}

\begin{abstract}
We present a case in which lung Ultrasound was relevant to reach an early diagnosis of lung tuberculosis and to manage the patient in the right setting. Moreover, ultrasound allowed to detect and treat massive pleural effusion through an ultrasound-guided thoracentesis.
\end{abstract}

Keywords: lung ultrasound, bedside, tuberculosis, pleural effusion.

\section{INTRODUCTION}

Tuberculosis (TB) is one of the leading causes of death due to infectious diseases, and it is a global health threat. TB incidence has undergone a rapid increase in the last decade, attributable to the increase in risk factors such as HIV co-infections, drug resistance and emigrations from areas where the disease is endemic. At today, TB is the major infectious causes of death in the world, with 1.6 million deaths in $2017^{1}$. Here, we present the case of a young policeman referred to our hospital for dyspnoea and respiratory failure. Ultrasound utilization allowed to reach a fast and specific diagnostic program, and to perform a diagnostic thoracentesis. Subsequently, we performed a narrative review on the role of ultrasound in diagnosing lung tuberculosis.

\section{CASE PRESENTATION}

A 36-year-old Caucasian policeman was referred to our Emergency Department for dyspnoea. He reported persistent cough and fever with night sweats for about 2 weeks, for which antibiotic therapy with Levofloxacin $750 \mathrm{mg}$ daily for 5 days was prescribed without clinical benefit. His past medical history was not suggestive for any disease. 
Blood pressure (BP) and cardiac rhythm were normal (BP: 130/75 mmHg; heart rate: $91 \mathrm{bpm}$, sinus rhythm), but the arterial blood gas analysis revealed a respiratory failure (peripheral oxygen saturation: $87 \%$ on room air; $\mathrm{pO}_{2}: 53 \mathrm{mmHg} ; \mathrm{pCO}_{2}: 41 \mathrm{mmHg}$ ). The physical examination showed a hypo-expansion of the left hemithorax, reduced tactile fremitus, medium-basal hypophonesis with vesicular murmur abolished.

Blood laboratory tests showed a blood glucose level of $60 \mathrm{mg} / \mathrm{dL}$ (normal value 74-106), a C-reactive protein of $15.10 \mathrm{mg} / \mathrm{dl}$ (n.v. 0.00-0.50), and the following blood count: white blood cells $10.760 / \mathrm{uL}$ (n.v. 4.00011.000), red blood cells 4.850.000/uL (n.v. 4.500.000-5.300.000), haemoglobin $14.2 \mathrm{~g} / \mathrm{dl}$ (n.v. 13-16,0), neutrophils $7.810 / \mathrm{uL}$ (n.v. 2.100-7.100), lymphocytes 1.800/uL (n.v. 1.100-3.000), monocytes 1.080/uL (n.v. 200-960), eosinophils 50/uL (n.v. 0-500), basophils 20/uL (n.v. 0 -200).

Urea, creatinine, sodium, potassium, calcium, transaminases, gamma-glutamyl transpeptidase, amylase, lipase, lactate dehydrogenase, total and fractionated bilirubin, and procalcitonin were normal.

Chest X-ray was required, and we performed a bedside lung ultrasound (LUS) to complete physical examination.

LUS showed a massive finely corpuscular anechoic pleural effusion with thin branches of fibrin on the left, with a consolidated lung parenchyma collapsing to the hilum as for complete atelectasis; in the context of the consolidated lung parenchyma, multiple round hypo-anechoic formations were seen, with finely irregular margins, not assuming colordoppler signal: cavitations? Abscesses?(Fig.1-2).

Therefore, chest X-ray confirmed the pleural effusion associated with consensual parenchymal hypoventilation and showed a widespread non-specific thickening of the bronchial walls. No pleural effusion on the right and no signs of pneumothorax were appreciable. The cardiac shape was within limits. Surprisingly, the radiographic images did not show the anechoic roundish formations identified by US.

A therapy with oxygen supplementation was set and the patient was admitted to the infectious diseases ward and isolated according to US findings.

In the ward, an ultrasound-guided diagnostic-evacuative thoracentesis was performed with drainage of yellow citrine liquid. Moreover, chest computed tomography (CT) was performed to better characterize the images observed with US and not confirmed by chest X-ray, thus showing an excavated formation with fluid-air levels in the apical segment of the left lower lobe of about $6 \times 3.5 \mathrm{~cm}$ (Fig.3). This formation was surrounded by an area of slight increase in the density of the pulmonary parenchyma, with ground-glass appearance.

Another subpleural area of parenchymal consolidation with pseudonodular morphology was present in the apicodorsal segment of the left upper lobe and appeared as excavated and communicating with the adjacent bronchus. A parenchymal consolidation area with appreciable subpleural morphology was present in the apical segment of the right upper lobe, showing a maximum axial diameter of about $2.2 \mathrm{~cm}$ and appeared excavated. Atelectasis striae in the lower segment of the lingula and in the basal pyramid of the left lower lobe were present. The findings described were therefore compatible with TB disease with cavitations. A fibrobronchoscopy was performed showing marked inflammation of the mucous membrane of the left bronchial hemisystem, where whitish secretions were detected.

The analysis of pleural fluid, bronchoalveolar lavage fluid and microbiological examination on sputum were positive for alcohol-acid resistant elements, confirming the diagnosis of pleuro-pulmonary TB. First-line specific antibiotic therapy was therefore set up and the patient was discharged on the seventh day in good clinical condition with indication for outpatient follow-up. The final diagnosis was pleuro-pulmonary cavitary tubercolosis.

\section{DISCUSSION \& LITERATURE REVIEW}

In past years, LUS has shown an increasing utilization in the diagnostic field alongside traditional diagnostic tests. Although the presence of an air interface below the pleura prevents the visualization of the lung in depth, the evaluation of sonographic artifacts allows to evaluate several pathological conditions and it is a useful tool to address a more focused and quickly diagnosis. 
To our knowledge, there are few data about US findings in lung $\mathrm{TB}^{2}$ focusing on the following fields of interest: detection of pleural effusion, assessment of residual pleural thickening, the execution of trans-thoracic needle biopsy, assessment of mediastinal lymphadenopathies and detection of pulmonary involvement in miliary $\mathrm{TB}^{3}$.

Sonographic pattern of pulmonary TB can be visualized as consolidations, subpleural nodules, pleural thickenings, fibrosis, pleural effusion and pneumothorax together with miliary pattern ${ }^{2,4,5}$. The miliary pattern has been described by Hunter et $\mathrm{al}^{6}$ as characterized by bilateral vertical artifacts (B-lines and/or "comettail artifacts") in multiple lung areas, and by sub-pleural granular changes, that may be more characteristic of miliary $\mathrm{TB}^{6}$. However, these findings are not so specific, since similar bilateral vertical artifacts have been described in patients with Pneumocystis jirovecii or cytomegaly virus pneumonia and sub-pleural US alterations can be found in other pulmonary conditions such as metastatic thyroid cancer ${ }^{6}$.

Agostinis et $\mathrm{al}^{2}$ classified the sonographic findings in patients with lung TB into two categories: lung (subpleural nodule, pleural effusion, miliary pattern and cavitations) and extra-lung (pericardial effusion, splenomegaly, abdomen lymph nodes, hepatomegaly, ascites) US findings ${ }^{2}$. The hypoecoic subpleural nodules (SUNs) were the most frequently identified in sixty adults with diagnosis of lung TB in a rural African setting $^{2}$. Consolidations were detected in about half of the patients and are indistinguishable from bacterial pneumonia, while cavitations were identified in three patients and described as anechoic or hypoechoic areas within solid lung consolidation ${ }^{2}$. SUNs were not identified in subjects without TB, suggesting a probable important role in lung TB diagnosis ${ }^{2}$.

The accuracy of LUS in the diagnosis of pulmonary TB in adults have been investigated in a recent study ${ }^{4}$. Montuori et $\mathrm{al}^{4}$ demonstrated that the combination of apical consolidations and SUNs found in the same patients has a specificity of $96 \%$ and a sensibility of $31 \%$, while when at least one of these signs is identified, the sensitivity and the specificity are respectively of 86 and $63 \%{ }^{4}$. Moreover, according to Agostinis et al ${ }^{2}$, cavitations were found to be the least common sign and not enough sensitive also in this study, probably due to the presence of air in cavitations or to the fact that a high number of lesions do not reach the pleura ${ }^{4}$.

In our case, the identification of the non-vascularized hypo-anechoic roundish lesions compatible with cavitations, in relation to the clinical context, was fundamental in the diagnostic suspicion of cavitary TB and for the early isolation of our patient in order to avoid the spreading of the infection in the crowded environment of the Emergency Room. It must be considered that our patient had not apparent risk factors for TB (patient medical history and lifestyle, HIV-negative test), and that the chest X-ray did not show the cavitations identified by US, so that the patient would have been hospitalized in a medical setting without the necessary isolation measures. Even if chest CT and microbiological and laboratory tests were fundamental in the different diagnosis between cavitations and lung abscesses, the findings provided by bedside LUS were relevant to confirm our diagnostic suspicion, and therefore for the instauration of an effective therapy, as already described in critical care ${ }^{7}$. In our case, we did not identify SUNs and apical consolidations because the lung parenchyma was completely collapsed due to the massive pleural effusion. However, the chest CT performed after evacuation of pleural fluid documented parenchymal consolidations at the level of the apical segments of the left upper and lower lobes, and of the right upper lobe, typical lung fields where consolidations can be found in post-primary tuberculosis ${ }^{8}$.

LUS is increasingly employed to safely perform bedside thoracentesis ${ }^{7,9-11}$. In our case, the possibility of identifying the corpuscular nature of the pleural fluid has contributed to the diagnostic suspicion ${ }^{5,12}$, and the US quantification of the fluid was fundamental to guide the therapeutic intervention ${ }^{13}$.

We did not perform a complete US evaluation of the abdomen since, in the Emergency setting and in the evaluation of the respiratory status of our patient who presented with a respiratory failure, we focused on the immediate evaluation of the lung parenchyma to diagnose the possible life-threatening conditions. However, the abdominal CT performed during hospitalization did not show lesions compatible with the dissemination of the disease.

Other possible applications of thoracic US in the diagnosis of TB are described in literature, especially in 
patients with suspected mediastinal TB (Table 1). Diagnosis of mediastinal TB is difficult due to non-specific clinical features and lack of characteristic radiographic features ${ }^{14}$. In order to obtain histopathological confirmation, a CT guided fine needle aspiration biopsy (FNAB) or invasive procedures such as mediastinoscopy or open surgical biopsy are often required ${ }^{14}$. US-guided FNAB has proven to be safe, and effective in the diagnosis of mediastinal $\mathrm{TB}^{14}$, despite its limitations for centrally located lesions due to the lack of a good acustic window ${ }^{15}$. More recently some authors have investigated the possibility of performing endobronchial ultrasound (EBUS) ${ }^{16,17}$, useful in investigating peripheral lung lesions, and also in guiding transbronchial FNAB and endoscopic aspiration from esophagus in suspected mediastinal $\mathrm{TB}^{18,19}$.

\section{CONCLUSIONS}

Bedside US is a safe, non-invasive, portable, versatile, easily repeatable and cost-effective imaging modality $^{4,7}$. Its use can lead to the identification of specific pleuro-pulmonary pathological conditions by strengthening the clinical-diagnostic suspicion. Although nowadays there are few data about LUS evaluation in tuberculosis, in our case it was fundamental in the formulation of diagnostic suspect through direct visualization of the characteristic lung lesions and early isolation of the patient; early suspicion allowed to promptly request the specific diagnostic investigations and begin the targeted therapy. Our case has also confirmed that LUS is important for the identification, quantization and characterization of pleural effusion and for the execution of diagnostic and therapeutic ultrasound-guided thoracentesis.

Informed consent was obtained for patient data publication

Declaration of interests

The authors declare that they have no known competing financial interests or personal relationships that could have appeared to influence the work reported in this paper.

No founding sources.

\section{REFERENCES}

1. Reid MJA, Arinaminpathy N, Bloom A, Bloom BR, Boehme C, Chaisson R, et al. Building a tuberculosis-free world: The Lancet Commission on tuberculosis. Lancet 2019; 393 (10178):1331-1384. DOI: $10.1016 /$ S0140-6736(19)30024-8

2. Agostinis P, Copetti R, Lapini L, Badona Monteiro G, N'Deque A, Baritussio A. Chest ultrasound findings in pulmonary tuberculosis. Trop Doct 2017; 47(4):320-328 . DOI:10.1177/0049475517709633

3. Di Gennaro F , Pisani L, Veronese N, Pizzol D, Lippolis V, Saracino A, et al. Potential Diagnostic Properties of Chest Ultrasound in Thoracic Tuberculosis - A Systematic Review. Int J Environ Res Public Health 2018; 15 (10). DOI: 10.3390/ijerph15102235

4. Montuori M, Casella F, Casazza G, Franzettia F, Pinib P, Invernizzi C, et al. Lung ultrasonography in pulmonary tuberculosis: A pilot study on diagnostic accuracy in a high-risk population. Eur J Intern Med 2019; 66 :29-34. DOI: https://doi.org/10.1016/j.ejim.2019.06.002

5. Heuvelings CC, Bélard S, Janssen S, Wallrauch C, Grobusch MP, Brunetti E, et al. Chest ultrasonography in patients with HIV: a case series and review of the literature. Infection 2016;44:1-10. DOI: https://doi.org/10.1007/s15010-015-0780-z

6. Hunter L, Bélard S, Janssen S, van Hoving DJ, Heller T. Miliary tuberculosis: Sonographic pattern in chest ultrasound. Infection 2015; 44 (2):243-246. DOI: 10.1007/s15010-015-0865-8

7. Bouhemad B, Zhang M, Lu Q, Rouby J-J. Clinical review: Bedside lung ultrasound in critical care practice. Crit Care 2007;11 (1):205. DOI: 10.1186/cc5668

8. Nachiappan AC, Rahbar K, Shi X, Guy ES, Mortani Barbosa Jr EJ , Shroff GS, et al. Pulmonary Tuberculosis: Role of Radiology in Diagnosis and Management. RadioGraphics 2017; 3 (1): 52-72. DOI: $10.1148 / \mathrm{rg} .2017160032$ 
9. Jones PW, Moyers JP, Rogers JT, Rodriguez RM, Lee YCG, Light RW. Ultrasound-Guided Thoracentesis: Is It a Safer Method? Chest2003; 123(2):418-423. DOI: 10.1378/chest.123.2.418

10. Feller-Kopman D. Ultrasound-Guided Thoracentesis. Chest 2006;129 (6):1709-1714. DOI:10.1378/chest.129.6.1709

11. Mercaldi CJ, Lanes SF. Complications and Improves the Cost of Care Among Patients Undergoing Thoracentesis and Paracentesis. Chest2013; 143 (2):532-538. DOI: 10.1378/chest.12-0447

12. Chen, H. et al. Sonographic Septation in Lymphocyte-Rich Exudative Pleural Effusions. J Ultrasound Med 25, 857-863 (2006).

13. Hew M, Tay TR. The efficacy of bedside chest ultrasound: from accuracy to outcomes. Eur. Respir. Rev. 2016;25 (141):230-246. DOI: 10.1183/16000617.0047-2016

14. Gulati M, Venkataramu NK, Gupta S, Sood BP, Sheena DM, Gupta SK, et al. Ultrasound guided fine needle aspiration biopsy in mediastinal tuberculosis. Int J Tuberc Lung Dis. 2000;4 (12):1164-1168.

15. Yuan A, Yang PC, Chang DB, Yu CJ, Lee LN, Wu HD, et al.Ultrasound guided aspiration biopsy for pulmonary tuberculosis with unusual radiographic appearances. Thorax 1993;48 (2):167-170. DOI:10.1136/thx.48.2.167

16. Chan A, Devanand A, Low SY, Koh MS. Radial endobronchial ultrasound in diagnosing peripheral lung lesions in a high tuberculosis setting.BMC Pulm Med 2015; 15, 90. DOI:https://doi.org/10.1186/s12890015-0089-9

17. Thangakunam B, Isaac BTJ, Christopher DJ. Endobronchial ultrasound experience in a high tuberculosis prevalence setting. Indian J Tuberc 2017; 64 (3):196-200. DOI:10.1016/j.ijtb.2016.11.035

18. Chalela R, Sánchez-Font A, Domínguez-Álvarez M, Badenes-Bonet D, Pijuan L, Curull V. Utilidad de la punción aspirativa transbronquial guiada con ultrasonografía endobronquial en el diagnóstico de la tuberculosis mediastínica. Med Clin (Barc).2016; 146(12):532-535. DOI:https://doi.org/10.1016/j.medcle.2016.07.022

19. Sharma M, Ecka RS, Somasundaram A, Shoukat A, Kirnake V. Endoscopic ultrasound in mediastinal tuberculosis. Lung India 2016;33 (2):129-134. DOI: 10.4103/0970-2113.177451

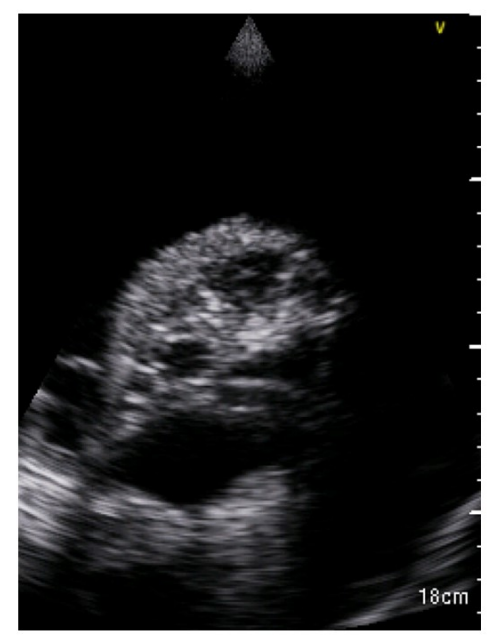

Fig.1 


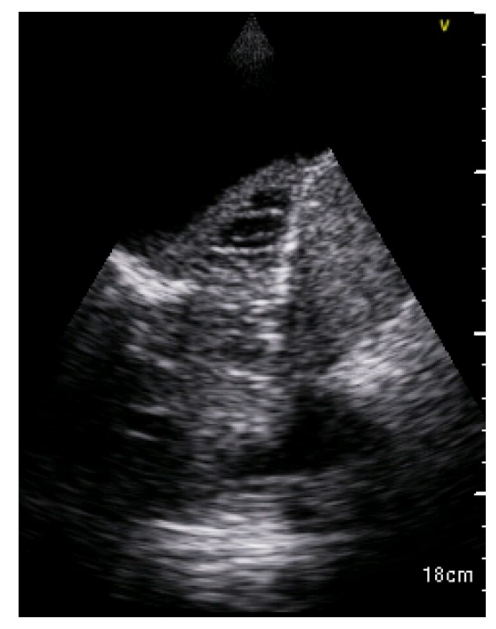

Fig.2

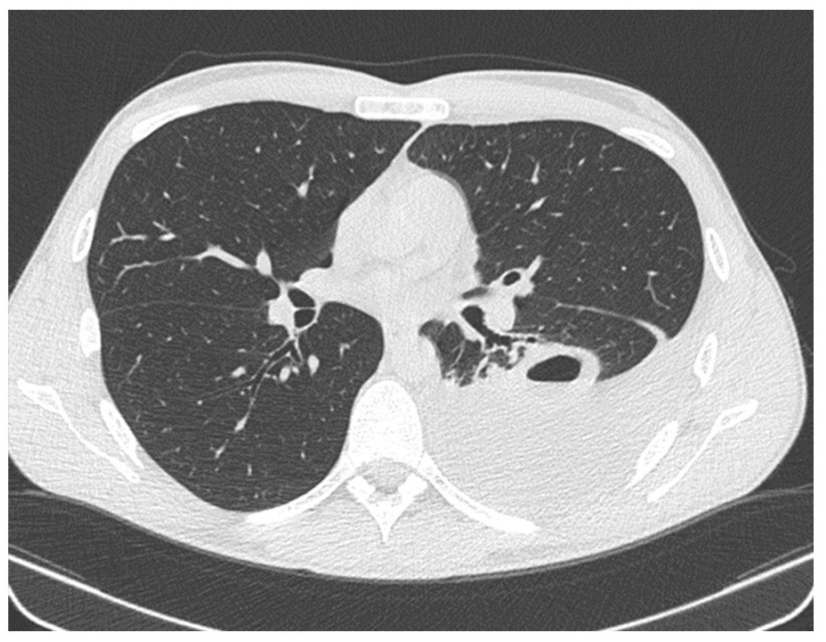

Fig.3

\section{FIGURE CAPTIONS}

Figure 1. Bedside thoracic US of the left hemithorax showing massive pleural effusion, complete lung atelectasis, and cavitations in the context of the consolidated parenchym.

Figure 2. Bedside thoracic US of the left hemithorax showing cavitations in collapsed left lung.

Figure 3. Chest CT after thoracentesis confirming the presence of excavated formation with fluid-air levels in the left lung. 
Agostinis $P$ et al. Trop Doct. 2017 [2]

Di Gennaro F et al.

Int $J$ Environ Res

Public Health 2018 [3]
60 adult patients

TUS

diagnosed with lung

TB

Review article

TUS
The most frequent finding was a SUN, which was mostly multiple and also found in radiologically normal areas. Other findings were lung consolidations, cavitations, miliary patterns made of miniature SUNs, and pleural and pericardial effusions. Chest US is a complementary tool in evaluating patients with suspected lung $\mathrm{TB}$ in resource-limited settings where the disease has high prevalence.

Five main fields of chest US application in TB were identified: (1) Detection, characterization, and quantification of TB;

(2) detection of residual pleural thickening after evacuation; (3) chest ultrasound-guided needle biopsy; (4) identification of pathologic mediastinal lymph nodes in children; and (5) identification of parenchymal ultrasound patterns. Effusion was also detected, in early stages, with signs of organization in $24 \%$ of patients. CUS was able to identify mediastinal lymph nodes in as many as $67 \%$ of patients with negative chest radiography. 
Montuori $M$ et al. Eur

J Intern Med. 2019 [4]

Patients admitted with

clinical suspicion of

PTB. PTB was

confirmed in 51 out of

102 patients.

Hunter $L$ et al.

Infection. 2016 [8]

Jones PW, et al. CHEST 2003 [9]

Feller-Kopman D. CHEST 2006 [10]

thoracentesis between

August 1997 and

September 2000

Review article
TUS

Multiple

consolidations, apical consolidations, superior quadrant

consolidations, and subpleural nodules were significantly associated with PTB diagnosis. Apical consolidation and subpleural nodules retained a significant association in a multivariate model, with an overall accuracy of 0.799 . B-lines and comet-tail artifacts disseminated throughout multiple lung areas and a pattern of sub-pleural granularity are seen in lung ultrasound of patients with pulmonary miliary TB diagnosed by chest radiography.

TUS

The complication rate with ultrasound-guided thoracentesis is lower than that reported for non-image-guided thoracentesis.
Ultrasonography is a portable and easily learned procedure that enhances the physical examination, and can provide real-time evaluation of the pleural space. It is becoming the standard of care for procedural guidance since its use has been associated with a reduction of complications due to thoracentesis. 
Mercaldi CJ and Lanes

SF. CHEST 2013 [11]

Chen HJ et al. J

Ultrasound Med. 2006

[12]

Hew M. and Tay T. Eur

Respir Rev 2016 [13]
Gulati $M$ et al. Int $J$

Tuberc Lung Dis. 2000

[14]

Yuan A et al. Thorax. 1993 [15]

Chan A et al. BMC

Pulm Med 2015 [16]
61,261 patients who had

a thoracentesis, including

26,838 (44\%) who had

US guidance.

73 patients with lung

cancer-related pleural

effusions and 93 with

tuberculous pleural

effusions

Review article

TUS

26 patients with a proven diagnosis of mediastinal TB

13 patients

TUS

TUS

TUS
TUS

EBUS

123 patients with

computed tomographic

evidence of PLLs who

underwent radial

EBUS guided

bronchoscopy
US-guided thoracentesis is associated with decreased risk of pneumothorax.

A complex septated pattern in the sonographic appearance is a useful predictor of tuberculosis in lymphocyte-rich exudative pleural effusions.

For bedside US of the pleura, there is evidence supporting diagnostic accuracy efficacy, and efficacy in guiding pleural interventions. Chest US for the lung parenchyma has an impact on diagnostic accuracy and decision-making for patients presenting with acute respiratory failure or breathlessness.

US guided FNAB is a safe, effective technique in the diagnosis of mediastinal TB. US can direct the needle to the most suitable part of a lesion to obtain the relevant specimens. The diagnostic yield is high and the procedure is relatively safe. It is especially helpful in patients with negative results of sputum and bronchoscopic examinations. EBUS-FNA is useful in investigating PLLs in a high TB incidence setting. Radial EBUS is a more rapid diagnosis technique for tuberculous lesions. 
Thangakunam B et al. Indian J Tuberc 2017

[17]

Chalela $R$ et al. Med Clin (Barc) 2016 [18]

Sharma $M$ et al. Lung India 2016 [19]
138 patients who EBUS

EBUS

patients with

EBUS

mediastinal

lymphadenopathy

without pulmonary

involvement

266 patients underwent

endoscopic ultrasound

guided fine needle

aspiration and 134

cases were diagnosed as

mediastinal

tuberculosis

EUS
In high TB prevalence countries, EBUS-FNA diagnoses a higher number of granulomatous than malignant diseases. EBUS-TBNA is a safe and effective technique in the diagnosis of patients with suspected mediastinal TB. EUS-FNA should be the investigation of choice for diagnosis of mediastinal tuberculosis.

Table 1. List and review of previously published works on the application of thoracic ultrasound in patients affected by TB.

Legend: US: ultrasound; TB: tuberculosis; TUS: thoracic ultrasound; SUN: subpleural nodule; PLLs: peripheral lung lesions; EBUS-TBNA: transbronchial needle aspiration guided by endoscopic ultrasonography; EBUS-FNA: endobronchial ultrasound-guided fine needle aspiration from bronchus; EUS-FNA: endoscopic ultrasound-guided aspiration from esophagus.

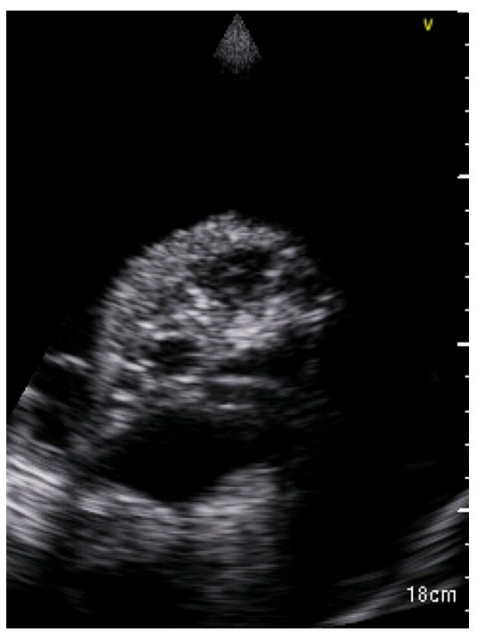



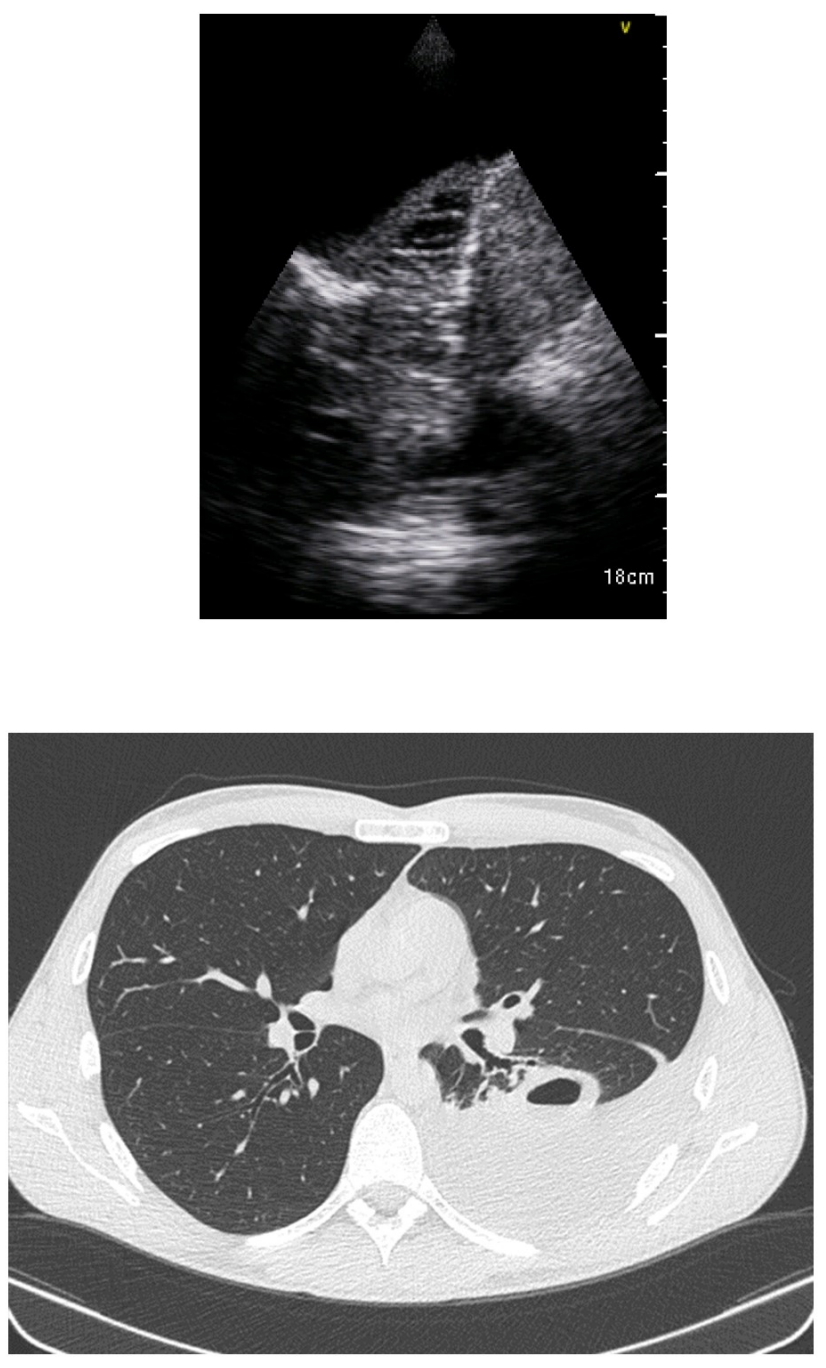